\title{
Lockdown Experiences of 10-13 Year Olds in New Zealand
}

\author{
Brigid McNeill,2,3 - Gail T. Gillon ${ }^{2,3}$
}

Received: 27 October 2021 / Accepted: 22 December 2021 / Published online: 29 December 2021

(c) The Author(s) under exclusive license to New Zealand Association for Research in Education 2021

\begin{abstract}
The implementation of lockdowns that include the closure of educational facilities for face to face teaching has been one of the strategies used internationally to contain the spread of the COVID-19 virus. Research suggests lockdowns are associated with negative impacts on children's psycho-social functioning, Most research, however, has been conducted in countries where extended lockdown periods have been in place and has primarily used parent/child survey to gain insight into lockdown effects. The current study was conducted in the context of New Zealand's initial 7-week national lockdown which allowed examination of the impact of a relatively short lockdown period. Participants $(n=139)$ aged 10 to 13 years from one school were interviewed face to face about their experiences during lockdown immediately following the re-opening of schools. Participants' self-concept was also evaluated to gain an understanding of their psycho-social skills after lockdown. Qualitative analysis identified positive and negative features of lockdown from children's perspectives. Analysis also focused on changes to children's relationships with close family members during lockdown. The findings have implications for identifying how to optimise lockdown experiences for children.
\end{abstract}

Keywords COVID-19 $\cdot$ Lockdown $\cdot$ Children $\cdot$ Wellbeing $\cdot$ Psycho-social functioning $\cdot$ Self-concept

Brigid McNeill

brigid.mcneill@canterbury.ac.nz

1 School of Teacher Education, College of Education Health and Human Development, University of Canterbury, Private Bag 4800, Christchurch 8140, New Zealand

2 University of Canterbury Child Well-being Research Institute, College of Education Health and Human Development, University of Canterbury, Christchurch, New Zealand

3 Better Start National Science Challenge, Liggins Institute, University of Auckland, Auckland, New Zealand 


\section{Introduction}

A key global strategy in response to the COVID-19 pandemic has been the implementation of regional or national "lockdowns" as a means of stopping the spread of the virus. Lockdowns involve severe restrictions on people's movements and have predominantly involved people staying within their place of residence and only exercising outside in their local area following specified rules for social distancing. Although lockdown is a term used across contexts, it is important to note that restrictions imposed during lockdowns differ from region to region. Most educational facilitates have been closed for face to face teaching during more stringent lockdown periods, however, with $91 \%$ of the world's student population adversely affected by such closures (Lee, 2020). Throughout the pandemic, the New Zealand Government has pursued an elimination strategy of the virus which began with a national lockdown of Covid Alert Level 4 and Level 3 restrictions from 23rd of March to 18th of May 2020 where schools and non-essential businesses were closed (Baker et al., 2020). This lockdown period also included two weeks of school holidays. People were also required to stay in their immediate household 'bubble' during Covid Alert Level 4 and Level 3. Although this initial national lockdown in New Zealand included severe restrictions on people's movement and socialising, it involved a relatively short period of school closures compared to that enacted in many other countries. The potential negative psychosocial consequences of lockdown for young children (e.g., social isolation, anxiety) has been highlighted in the literature (Jiao et al., 2020). Given the relatively short nature of this initial period of school closures in New Zealand, however, the impact on children's psycho-social functioning may not be as marked. The purpose of this study is to describe how a group of New Zealand children experienced the first national lockdown alongside an objective measure of their selfconcept immediately following the re-opening of schools. The findings from the study have important implications for supporting children through disruptions caused by the COVID-19 pandemic.

\section{Lockdowns and Children's Psycho-social Functioning}

There has been growing attention in the literature to the potential negative effects of school closures and other lockdown restrictions on children's psycho-social functioning. Singh et al. (2020) conducted a narrative review of research and advisory papers regarding the impact of COVID-19 and lockdowns on the mental health of children and adolescents. The review highlighted concerns related to four main areas: children's education, support of children with special learning needs, impact on children from lower socioeconomic backgrounds and the impact of separation from parents due to isolation procedures following exposure or infection. In all, the review showed that school closures result in a range of factors that can negatively affect children's psycho-social functioning. These factors 
include a change in routine, increased stress in the family, separation from friends and social networks, and boredom. Other authors have also emphasised disruption in extracurricular activities, limited contact with friends and unstructured schooling time which can all contribute to higher rates of anxiety in children and young people (Jiao et al., 2020; Liu et al., 2020).

Panda et al. (2021) conducted a systematic review regarding the psychological and behavioural impact of lockdown and quarantine measures for children and adolescents. The review included 15 studies across multiple international contexts (e.g., India, China, the Netherlands, Italy, Spain, etc.). The majority of studies utilised parent report via survey to ascertain the impact of lockdown and quarantine measures on children. Over $30 \%$ of children and adolescents across studies exhibited features of anxiety, depression, irritability and attention difficulties. Boredom and disrupted sleep patterns were also evident at high rates. Children who appeared most affected had a behaviour or psychological difficulty prior to lockdown starting. Negative psycho-social impact appeared to be more pronounced in adolescents over 13 years. Further, there were geographic differences in outcomes with children from Asian countries exhibiting more pronounced negative impacts of psycho-social functioning than children from western counties. The review highlights the potential negative consequences of lockdown periods on children's well-being but cautions against assuming impacts will be equivalent across geographic contexts and age groups.

There is emerging evidence that younger children may be particularly impacted by the negative effects of lockdown periods in some contexts. Waite et al. (2021) surveyed 2673 parents and carers in the UK at two points during lockdown (March and May 2020) regarding the mental health of their children aged between 4 and 16 years. Younger children (4 to 10 years) experienced more pronounced changes in psycho-social functioning across the two survey points than adolescents. For example, there was a 10 to $35 \%$ increase in emotional, inattention and conduct symptoms for younger children compared to a 4 to $8 \%$ increase in adolescents. Children and young people from lower socioeconomic communities and those with special educational needs had more pronounced emotional and behavioural symptoms at both time points.

Achterberg et al. (2021) examined lockdown effects in 10-13 year olds in the Netherlands. The longitudinal study (which had started before lockdown) examined the link between parents' stress levels, coping strategies and children's behaviour. The children's longitudinal data showed an overall decrease in externalising and internalising behaviours over time (including during the lockdown). Further, there was a generally low level of parental and child stress during lockdown. The decrease in children's negative behaviours over the lockdown period, however, was less than expected suggesting that this period may have had prompted a slowing in some features of children's behavioural development. Analyses showed an interaction between parental stress levels, family coping strategies and children's behaviour over time. Children whose parents had a more over-reactive parenting style before lockdown were more likely to experience negative consequences during lockdown. Further, parents and children who exhibited lower wellbeing before lockdown reported lockdown being more stressful which decreased wellbeing further. The authors, however, also reported some positive implications of the lockdown period. 
For example, many participants reported that they enjoyed having more family time. Analyses that identify positive and protective factors (in addition to negatives and risk factors) provide critical information for directing how children's and family's resilience can be built during this challenging time (Masten \& Motti-Stefanidi, 2020).

Mangiavacchi et al. (2021) also highlighted the critical role that parents and home learning can play in influencing children's wellbeing during lockdown. This study surveyed Italian parents with children under 16 years during lockdown. Topics in the survey were children's wellbeing, home learning, and household responsibilities during lockdown. Analyses revealed extra involvement of fathers in childcare and home schooling during lockdown was associated with wellbeing benefits for children. Further, the overall negative impact of lockdown on children's wellbeing was mitigated by features of home learning (particularly access to live online learning opportunities), having other children in the household, having well educated parents and having both parents at home during lockdown.

Given one of the key changes for children during lockdown is the commencement of online or home learning, it is not surprising that this factor can be a key contributor to children's psycho-social functioning. Brom et al. (2020) surveyed Czech parents regarding online schooling their children aged 6 to 15 years $(n=9810)$. Children spent an average of two to four hours a day engaged in learning. Parents' biggest concerns about online learning was the lack of time to support children, problems with technology and lacking knowledge regarding how to teach and/or teaching content. Sancho et al. (2021) also included a focus on home learning in their survey of Spanish parents regarding 1225 children's (aged 2-12 years) wellbeing during lockdown. One of parents' biggest concerns were related to children's home learning, particularly in regard to feeling that children were set too much online learning. Although these initial studies focused on parental report regarding online learning during lockdown provide valuable information, it is also important to gain the perspective of the children themselves. It is clear that online learning can act as a protective or risk factor for children's psycho-social functioning during lockdown. Understanding children's experiences may thus play a crucial role in optimising its function.

\section{Children's Self-concept as an Important Element of Psychosocial Functioning}

Studies focused on exploring psycho-social impacts of lockdowns on children have focused broadly on aspects of children's behaviour and wellbeing. Alongside the importance of including children's experiences, a specific focus on their self-concept as a component of psycho-social functioning may also elucidate more about children's wellbeing from their perspective following lockdown. Self-concept is an individual's belief and knowledge about themselves that can be assessed across a variety of domains (e.g., academic, behavioural, social etc.). Self-concept has been studied extensively in educational settings due to its relationship with academic outcomes, making it a useful construct to focus on with children. Self-concept is also 
related to many of the negative psychological and behavioural consequences of lockdowns reviewed in the literature above. For example, Ybrandt (2008) examined the relationship between self-concept and internalising and externalising behaviours in 227 adolescents. The analyses showed that a positive self-concept was the primary protective factor for adjustment and positive behaviour. On the other hand, negative self-concept was a prominent risk factor for problematic internalising behaviours in girls. There was also a strong relationship between negative self-concept and externalising behaviour, particularly in older boys. It follows that having a focus on self-concept is related to broader aspects of psycho-social functioning and children's well-being (Pfeifer \& Bergman, 2018) whilst also maintaining a focus on the perspective of the child.

Studies that have examined children's self-concept in relation to lockdowns is scarce in the literature. Lindell-Postigo et al. (2020) examined self-concept of 136 Spanish adolescents (12-18 years) in relation to their physical activity during lockdown. The results suggested that children. It should be noted, however, that the study was cross-sectional in nature and the finding of lowered self-concept was reliant on comparing to previous studies rather than monitoring change in this cohort. Children's broader experiences of lockdown (beyond physical activity) was not addressed in the study. González-Valero et al. (2020) examined adolescents"' selfconcept before and during lockdown in relation to their physical activity. The results showed that the cohort had lower general, social and emotional self-concept during lockdown than before lockdown. Participants had stronger academic self-concept during lockdown. The authors attributed this finding to the greater level of schooling support provided in the home during the lockdown period. Further examination of children's self-concept during lockdown periods that takes into account their experiences is needed.

\section{Children's Experiences of Lockdown in New Zealand}

Emerging accounts of the negative psycho-social impacts of lockdowns for children have come from countries that have experienced long periods of school closures and have primarily relied on parental report. Contrastingly, in New Zealand the relatively short initial 7 week lockdown period appears to be associated with more muted negative impacts on children's psycho-social functioning. New Zealand's Office of the Children's Commissioner (2020) distributed an online survey focused on children's lockdown experiences in the week's following the re-opening of schools. The survey was completed by 1402 children and young people aged 9 to 18 years and was focused on children's education, relationships, and wellbeing during lockdown. The survey sought to identify both positive and negative influences on wellbeing for children and youth during the lockdown period.

The findings showed that although most respondents had relatively positive experiences during lockdown, a significant group had negative experiences. There were positive and negative impacts on children's relationships with their parents, sibling and friends during lockdown. The overarching theme was children valuing having more time to spend and connect with their family. Children also valued having 
autonomy in how to structure daily activities but online learning brought challenges for many. The report highlighted that children from lower socioeconomic backgrounds were more likely to have negative experiences during lockdown and have lower wellbeing ratings. Finally, the authors highlighted the over-representation of respondents from high socioeconomic communities in the study.

The mixed negative and positive experiences for adults during the first national New Zealand lockdown have also been highlighted. Every-Palmer et al. (2020) utilised an online survey to investigate the psychological impact of New Zealand's initial lockdown on adults. The survey included three standardised assessments of psychological functioning and wellbeing alongside other items designed to probe additional negative and positive aspects of lockdown. Results showed around a third of participants reported moderate to severe psychological distress and almost $40 \%$ of respondents had a low wellbeing score. Younger adults (aged 18-34 years), those with mental health illness prior to lockdown, and those who had experienced employment issues were more likely to present with psychological issues and/or lower wellbeing. Positive features of the lockdown included more family and home time and less pollution. Although the study was not focused on children, it has important implications for children's wellbeing given the critical role that parents play in mediating their children's risk of negative psycho-social consequences from lockdown (Achterberg et al., 2021; Mangiavacchi et al., 2021).

The Children's Commissioner's report provides a summary of New Zealand children's experiences during lockdown. The online survey format, however, where respondents needed to independently read and write their answers may have limited its accessibility to children with lower levels of literacy, particularly the younger children in the sample. Another factor to consider when interpreting the findings of the Children's Commissioner's report is the broad age range included in the sample. Given associations between lockdown experiences and wellbeing appear to differ across age groups (e.g., Waite et al., 2021), it is important to look into the experiences of a particular age bracket. The transitional period from childhood to adolescence, for example, is an important period to study given the social reorientation and heightened emotional responsivity that tends to occur during these years (Orben et al., 2020).

\section{The Current Study}

Overall the literature suggests that lockdowns related to COVID-19 can have a range of negative impacts on children's wellbeing and psychosocial functioning. The majority of studies in this area, however, have utilised parental report (via survey) to gain insight into children's experiences. Previous work has also tended to focus on a wide age-range of children and has not included direct measurement of aspects of psycho-social functioning to supplement survey data. Finally, it is evident that the impact of lockdowns is varied across geographic contexts, meaning it is important to have further insight into the experiences of New Zealand children rather than relying on the international literature. The current study is unique in terms of addressing these gaps in the literature. 
To supplement findings from large survey data, the current study sought to document the lockdown experiences of New Zealand students from one intermediate school (i.e., children aged 10 to 13 years) via a face to face interview. As highlighted in the review above, this age group may have particular social and emotional consequence of lockdown experiences. At the time of the interview, students also completed a formal measure of self-concept so that some insight into the cohort's psycho-social functioning immediately post-lockdown could be gained in addition to hearing their story.

In particular, the study sought to answer the following research questions:

- What were participants' negative and positive experiences during New Zealand's initial national COVID-19 lockdown?

- How did participants' relationships with people in their bubble change during the lockdown period?

- What was the cohort's self-concept as measured by a normative tool immediately following New Zealand's initial national COVID-19 lockdown?

\section{Materials and Methods}

\section{Study Design}

The study employed a mixed-methods design to address the research questions. Qualitative data (interview data) was collected and analysed alongside quantitative data (children self-concept) to gain an understanding of children's experiences and their self-concept immediately following a lockdown period.

\section{Participants}

All children attending an Intermediate School (Year 7 and Year 8) in Christchurch, New Zealand were invited to participate in the project. This study was part of a broader project focused on examining the links between progress in literacy learning and children's psycho-social functioning before the transition to the secondary schooling context. The school was thus recruited through convenience sampling to gain understanding of children's experiences during the lockdown. The school has a decile ranking of 7 (suggesting an average socio-economic area) but draws students from a wide area that includes over 45 contributing primary schools across the city.

From the 240 students invited to participate 139 students ( 74 males, 65 females), or $59 \%$ of those invited provided informed consent in accordance with ethics approval (parental and student consent) to participate in the study. There were 61 Year 7 students (Mean age $=11.6$; SD =0.34) and 78 Year 8 students (Mean age $=12.6 ; \mathrm{SD}=0.37)$ in the cohort. The ethnicity of the cohort was: New Zealand European (63.1\%), Māori (11.3\%), Pacific (4.3\%), Asian (8.5\%), Other (12.8\%). Students who used English as an additional language comprised $8.5 \%$ of the cohort. 


\section{Measures and Procedure}

All interviews took place in a 15 min session which was led by a Speech and Language Therapist (SLT). Participants were seen individually in a quiet space at the school. All sessions were audio-recorded for transcription and reliability purposes. The session took place in the first four weeks following schools re-opening after the March 2020 lockdown.

\section{COVID-19 Interview}

The interview protocol included posing three questions to the participants which related to their experiences in lockdown. The questions (in order) were:

(1) Tell me what was the best thing about staying at home during the lockdown.

(2) Tell me what you didn't like as much about staying home during the lockdown.

(3) Tell me about any changes in how you got on with the people in your bubble during the lockdown?

The SLT asked each participant the questions as presented above. The protocol dictated that only non-specific prompts were used to encourage the participant to talk more (e.g., can you tell me more about that?). If the participants were nonresponsive or struggling to think of an answer these non-specific prompts were used to encourage the student's responses. If students did not response after being prompted twice, the next question was presented.

\section{Analysis}

An inductive thematic analysis of the COVID-19 interview data was conducted to analyse the responses to each COVID-19 interview question. The thematic analysis included open, axial and selective coding processes to ensure the validity of the analysis (Strauss \& Corbin, 1990). Initially, the first author and a research assistant independently open coded the responses to the three questions. The first author then categorised codes from the independent analysis based on discussion with the research assistant regarding the similarities and differences that had occurred in the open coding process. Axial coding was then used by the first author and research assistant independently where these new codes were applied to the interview data followed by comparison and discussion to refine the coding categories further. This axial coding process was undertaken for $50 \%$ of the interview data. Axial coding resulted in the identification of 16 main parent codes for the first question with 4 containing 2-3 more precise codes, 8 main parent codes for the second question and 12 main parent codes for the third question with 5 containing 2-3 more precise codes. In the final stage of analysis, selective coding was used by the first author who reviewed the interview data a final time and coded into overarching themes identified through the axial phase. Quotes from 
the dataset were included in the reporting of results to enhance trustworthiness of the data analysis (Elo et al., 2014).

The following measures were included to ensure trustworthiness of the data (Elo et al., 2014; Shenton, 2004): recruiting participants from entire school roll, independent coding procedures by two coders in the process to identify thematic analysis, triangulation of data (across participants and connection to self-concept data), framing findings within the literature base and in-depth description of methodology that allows replication.

\section{Piers-Harris Self-concept Scale (Piers et al., 2018)}

This normative, self-reported measure of self-concept was administered to gain an objective measure of one aspect of participants' psycho-social functioning following the lockdown period. In this assessment, participants answered 58 yes or no questions related to themselves (e.g., I hate school, I am a good person, I forget what I learn). These questions then provided a score for six different domains: Behavioural adjustment, Freedom from anxiety, Happiness and Satisfaction, Intellectual and social status, Physical appearance and attributes and social acceptance. The tool is written at a first grade reading level so could be accessed by the majority of the cohort. For children who needed support to read the questions, these were read to the student so they could provide an answer.

\section{Analysis}

The following scores were collected from the Piers-Harris Concept Scale. For all scores, a score in the low range (less than 39) or the very low range (less than 29) was characteristic of students who may require more in-depth psychosocial assessment and/or would benefit from support.

- Total score which provided a measure of general self-concept.

- Behavioural adjustment score which provided a measure of disclosure or denial of difficult behaviour. Children scoring in the average range on this component (40-55) describe themselves as generally well behaved whilst showing some awareness of their difficult behaviours.

- Freedom from anxiety score which provided a measure of anxiety level.

- Happiness and satisfaction score which provides a general measure of happiness.

- Intellectual and school status score which provides a measure of the student's evaluation of their intellectual and academic skills. Scores in the lower ranges for this component indicates the student finds school difficult and has a low perception of themselves as a learner.

- Physical appearance and attributes score which provides a measure of the student's perception of their appearance and attributes such as leadership. A score in the lower ranges on this component indicates that the student has poor body image and feel they are not well regarded by peers. 
- Social acceptance score which provides a measure of the student's perception of their social skills. Scores in the lower ranges on this component indicate that the student feels dissatisfied with their social skills.

All response forms were re-scored by an independent assessor for reliability purposes with $100 \%$ agreement across examiners.

\section{Results}

\section{COVID-19 Interview}

The main themes for each of the three questions within the COVID-19 were identified along with quotes to exemplify the primary themes. The first question asked students to identify the 'best thing' about the lockdown period. The most commonly reported positive aspect of lockdown for participants was sleeping in (reported by 46 of the 141 participants). Other common positive themes to emerge from the interview data for this question included spending time with family $(n=37)$, having screen time (e.g. playing games online, watching movies) $(n=35)$ and having less school work to complete $(n=22)$.

Quotes from participants in relation to positive experiences during lockdown included:

Sleeping in til 2! I never get to sleep in like that cause my dad would always wake up at about six so he can go to work, but cause he wasn't going to work then he would also sleep in for ages.

We were able to choose a time when we do our schoolwork instead of having to do it. And we got longer times to do it [school work] instead of having to rush and not do our best work.

The best thing was probably just spending more time with my family, cause most of the time they're at work.

I went for walks regularly. And there are a lot of people putting teddy bears in their windows around the community. So I thought that was quite nice. Seeing people doing chalk drawings on the driveways and just people genuinely being a lot more friendlier and coming together as a community was quite nice.

The full list of primary themes that were identified and their frequency of use can be viewed in Table 1.

The second interview question asked students to identify aspects of lockdown that did not like. The most commonly reported negative response about lockdown was missing friends (reported by 77 out of 141 participants). Other common negative themes include being bored $(n=31)$, feeling stuck at home $(n=27)$ and online/home schooling $(\mathrm{n}=27)$.

Example quotes from participants in relation to negative lockdown experiences: 
Table 1 Themes regarding participants' positive experiences of lockdown

\begin{tabular}{lcc}
\hline Theme & Count & Percentage \\
\hline Sleeping in & 46 & 33.1 \\
Spending time with family & 37 & 26.7 \\
Screen time (e.g., playing games, television, movies, online.) & 35 & 25.2 \\
Not going to school or having less school work to do & 22 & 15.8 \\
Not being rushed or busy & 20 & 14.4 \\
Going for walks & 15 & 10.8 \\
More a more flexible routine/timetable for school & 14 & 10.1 \\
Chatting with friends online & 10 & 7.2 \\
Home/online schooling & 9 & 6.5 \\
Quiet indoors activity (writing, art, reading) & 6 & 4.3 \\
Playing with pet & 4 & 2.9 \\
No uniform & 4 & 2.9 \\
Other & 4 & 2.9 \\
Making food, baking & 3 & 2.2 \\
\hline
\end{tabular}

Well, you didn't get to see your friends, which really sucked. I'm one of those people who likes company. I like, just playing games with people and socializing.

There was like no freedom, couldn't go anywhere.

Well, learning was a bit hard. Like long division, I didn't really understand it. .....the teacher tried to explain it to me. But I still don't really get it, it's easier to explain it, when you are not on text or computer.

The full list of themes related to this prompt that were identified and their frequency of use can be viewed in Table 2 .

The third question prompted participants to talk about any changes in how they got on with the people in their bubble during the lockdown. The most commonly

Table 2 Themes regarding participants' negative experiences of lockdown

\begin{tabular}{lll}
\hline Theme & Count & Percentage \\
\hline Missing my friends & 77 & 55.4 \\
Being bored & 31 & 22.3 \\
Didn't get to go anywhere & 27 & 19.4 \\
Home schooling/online schooling & 23 & 16.5 \\
Couldn't go to sports & 19 & 13.7 \\
Not getting on with/annoyed by siblings & 19 & 13.7 \\
Missing extended family & 12 & 8.6 \\
Not getting on with/annoyed by parents & 5 & 3.6 \\
No takeaways & 4 & 2.9 \\
Chores & 4 & 2.9 \\
Other & 4 & 2.9 \\
\hline
\end{tabular}


reported theme was no change in how they got on with their parents and/or siblings $(n=46)$. Other highly reported themes included having more time together $(n=24)$, getting on better with parents/stepparents $(n=24)$ and being able to talk more with parents/stepparents $(n=17)$.

Example quotes regarding this question are:

We got along better than what we used to because we got to spend more time together.

I spent a lot more time with them [parents], I like opened up to them about a lot of things like - my emotions and stuff like that, which I usually wouldn't do. I feel like we've got a better connection because normally we don't talk as much but now we're like, really close.

I'm now good friends with my mum's boyfriend's son cause he's real funny, I found out that during lockdown.

The full list of primary themes that were identified and their frequency of use can be viewed in Table 3 .

\section{Self-concept}

To gain an understanding of the cohort's self-concept, the average score (and standard deviation) for each component within the Piers-Harris was calculated. The results show that participants' scores were within the expected range on average for all components of the assessment. The self-concept scores thus appear to align with the relatively positive perception many participants had regarding their lockdown experience (e.g., the majority of participants reported either no change or getting along better with family members during the lockdown. These scores are presented in Table 4 below.

In order to understand the proportion of children that may need support and/or further in-depth evaluation of their self-concept, the numbers of students within the

Table 3 Themes regarding changes in participants' relationships during lockdown

\begin{tabular}{ll}
\hline Theme & Count \\
\hline No change & 46 \\
Spent more time with family & 24 \\
Got on better with parent/stepparent & 22 \\
Talked more & 17 \\
Family walks and bike rides & 16 \\
Changes as working/studying at home & 12 \\
Fighting more with siblings & 10 \\
Parents more grumpy & 10 \\
Getting on better with siblings & 10 \\
More arguments in family & 9 \\
Family got on well & 8 \\
\hline
\end{tabular}


Table 4 Average scores in the components of the Piers Harris Assessment (Piers et al., 2018)

\begin{tabular}{lcc}
\hline Sub-test & Mean & $\begin{array}{c}\text { Standard } \\
\text { deviation }\end{array}$ \\
\hline Behavioural adjustment & 50.1 & 8.7 \\
Freedom from anxiety & 48.6 & 10.2 \\
Social acceptance & 50.0 & 8.6 \\
Happiness and satisfaction & 46.6 & 10.8 \\
Intellectual and schooling status & 48.7 & 8.9 \\
Physical appearance and attributes & 46.3 & 8.8 \\
Total score & 46.8 & 8.0 \\
\hline
\end{tabular}

Note Scores between 40 to 55 are considered average on this tool

Table 5 Percentage of participants in each score range for the components of the Piers-Harris Assessment (Piers et al., 2018)

\begin{tabular}{llllc}
\hline Sub-Test & High $(>55)(\%)$ & $\begin{array}{l}\text { Average } \\
(40-55)(\%)\end{array}$ & $\begin{array}{l}\text { Lower } \\
(30-39)(\%)\end{array}$ & $\begin{array}{l}\text { Lowest } \\
(<29) \\
(\%)\end{array}$ \\
\hline Behavioural Adjustment & & 37.4 & 13.7 & 1.4 \\
Freedom from anxiety & 47.5 & 48.9 & 18.7 & 2.2 \\
Social acceptance & 30.2 & 44.6 & 20.9 & 5.8 \\
Happiness and satisfaction & 28.8 & 48.2 & 7.2 & 6.5 \\
Intellectual and schooling status & 44.6 & 29.5 & 13.7 & 12.2 \\
Physical appearance and attributes & 38.1 & 45.3 & 12.9 & 3.6 \\
Total Score & 15.1 & 67.6 & 16.5 & 0.7 \\
\hline
\end{tabular}

high, average, lower and lowest ranges for each of the components of the Piers-Harris was calculated (see Table 5). Results showed that the majority of the cohort were in the high or average ranges, but a significant proportion exhibited scores in the lower and lowest ranges. Descriptive analysis of this data shows that more participants in the low ranges for the 'intellectual and schooling status' and 'social status' components of the self-concept assessment. In the discussion section, potential connections between participants' lockdown experiences and their self-concept in particular components are further explored.

\section{Discussion}

National and regional lockdowns in response to the COVID-19 pandemic have been one of the common global strategies to reduce the spread of the virus. These lockdown periods have been associated with negative psycho-social impacts on both children and adults internationally. The virus elimination strategy led by the New Zealand government has meant that lockdowns have had high levels of social restrictions but have been relatively short. This study sought to document what children 
did and did not enjoy during lockdown for pre-adolescents from one intermediate school in a main city. The cohort's perceptions about their relationships with people in their "bubble" (i.e., immediate household) during lockdown were also documented. Finally, the study captured students' self-concept via the administration of a formal assessment in the four weeks after schools were re-opened for face to face teaching.

Participants' most commonly reported positive features about lockdown were associated with: (1) their ability to schedule their day more independently (through being able to sleep in, having less school work and having more autonomy over their school timetable), (2) having more time to spend with family and (3) increased access to screen time for entertainment and connection with friends. These results align strongly with other positive features of lockdown for children have been reported in the literature (Achterberg et al., 2021; Office of the Children's Commissioner, 2020), particularly in regard to children enjoying the increased time to spend with family and having more control over their daily schedule. These findings represent one of the few accounts of the positive consequences of lockdown from children's perspectives. Understanding features of lockdown that children find positive is important information that could help parents and teachers shape the lockdown environment in a way that is more supportive for young people. This perspective is also in line with a recent call to examine positive and negative consequences of lockdown for children and families in order to offer a more balanced view within the literature (Bruining et al., 2020).

The most commonly reported positive aspect about lockdown overall was sleeping in. Within the literature, sleeping in itself is not a commonly reported positive feature of lockdown from children's perspectives (beyond having more freedom regarding the day's activity). This feature may be particularly salient for this cohort given they were all in the pre-adolescent age group. The finding highlights the benefits of looking at particular age groups in isolation when examining the impact of lockdown as has been reported in previous studies (see, Waite et al.,2021).

The most common aspects about lockdown that participant did not enjoy were related to a lack of connections with friends, boredom and online learning. The literature has shown that such negative experiences can impact negatively on wellbeing and behaviour, particularly for children within families with more limited coping strategies, children from lower socioeconomic communities, or children who have lower wellbeing prior to the onset of lockdown (Achterberg et al., 2021; Panda et al., 2021; Singh et al., 2020). The results suggest that strategies such as supporting children to connect with friends electronically and enhancing pedagogy and accessibility of online learning would be beneficial to enhance children's experiences during lockdown. Particular strategies that may be useful to optimise online learning include having more 'live' online lessons (Mangiavacchi et al., 2021) and providing additional support to parents who are trying to support children in their learning at home (Brom et al., 2020; Sancho et al., 2021).

The majority of participants reported no change in their relationships with people in their bubble during lockdown. However, a large proportion also reported positive changes to relationships. Positive impacts on children's relationships were primarily related to the opportunity to spend more time together and particularly to talk more. 
Finding ways to ensure that families can have more time together to talk during lockdown is a potential protective factor for children's wellbeing during lockdown. Of course, finding such time to connect is more difficult for families where both parents are working from home and where stress is at a higher level within the household (Achterberg et al., 2021). The findings are also a reminder of the importance of finding time to talk with children at any time, particularly for children in the pre-adolescent stage when they are re-orienting socially and starting to see parents and siblings in a different light.

Although the majority of children had generally positive experiences during lockdown and the majority of their relationships stayed the same or improved, it must be highlighted that a significant proportion of the cohort also experienced negative experiences and/or their relationships were negatively impacted. Although not directly measured in the current study, the literature review suggests that children who have negative psycho-social impact in response to lockdown are more likely to be those families and children with pre-existing difficulties, families with fewer resources and families where pre-lockdown stress levels are heightened. It is important that specialist support continues for children and families during lockdown (albeit via distance) and that it is easy for children and families to reach out for a new service if they are not coping with the extra demands of lockdown.

The examination of the Piers-Harris data showed that the cohort generally had self-concept within the average or high range immediately following the lockdown experience. There was only one participant who fell into the 'lowest' category on the total self-concept score. This data is in line with that reported through the wellbeing portion of the New Zealand's Children's Commissioner's report (2020). Although a causal connection between children's self-concept as measured by the administered assessment and their lockdown experiences can not be made, there are interesting associations between particular sub-constructs of self-concept and the themes identified about participants' experiences through the interview data. In the 'intellectual and schooling status' and 'social status' categories, there were 36 and 37 participants respectively who fell into either the low or lowest range (suggesting potential need for further assessment and/or support). This was compared to the other subconstructs where there were 19 to 29 participations identified in the low or lowest range. Within the interview analysis, difficulties with remote learning and feeling disconnected from friends were two prominent themes that are consistent with the cohort appearing to have more children with low self-concept within these areas. It is also intuitive that children may not rate themselves as highly on social status after a period of limited interaction outside of their bubbles. Further research that includes data points over time (before, during and after lockdown) is important to shed light on the potential link between children's lockdown experiences and particular areas of psycho-social functioning and/or self-concept.

\section{Limitations}

Although this study is novel given its focus on children's interview data regarding lockdown experiences alongside a direct measure of self-concept, there are limitations that should be taken into account when interpreting the findings. Participants 
were located in one intermediate school. Although the students enrolled in this school came from 46 contributing schools from across the city, the generalisability of the findings is limited. The cross-sectional nature of the study means that direct information regarding changes to children's self-concept over time in response to their lockdown experiences can not be gleaned from these results.

\section{Conclusion}

The findings showed that pre-adolescent children had both positive and negative experiences during the initial New Zealand national lockdown in response to the COVID 19 pandemic. The shorter lockdown period may have had a limited negative impact on students' self-concept immediately following the lockdown although further research utilising a pre-post design is required to more fully investigate this relationship. These results are in contrast to the predominantly negative psychosocial impacts that have been reported across other international contexts (Panda et al., 2021). It may well be that repeated lockdown experiences for children in New Zealand may have longer term negative impacts as the country continues its strategy to protect the community.

The study's methodology of complementing children's voice with direct assessment of self-concept provided useful data to more fully understand the perceived impact of the lockdown on children from their perspectives. Protective factors that are related with a more positive lockdown experience include children having autonomy over their time, ensuring families have resources that enable them to have more time with their children, and supporting children to maintain connections with their friends from a distance.

The findings provide an important reminder of the value of listening to children's voices as we navigate how best to support children, young people and their families through the COVID-19 environment.

Funding The funding was provided by Ministry of Education-New Zealand

\section{References}

Achterberg, M., Dobbelaar, S., Boer, O. D., \& Crone, E. A. (2021). Perceived stress as mediator for longitudinal effects of the COVID-19 lockdown on wellbeing of parents and children. Scientific Reports, 11(1), 1-14.

Baker, M. G., Kvalsvig, A., Verrall, A. J., \& Wellington, N. (2020). New Zealand's COVID-19 elimination strategy. The Medical Journal of Australia, 213(5), 198-200.

Brom, C., Lukavský, J., Greger, D., Hannemann, T., Straková, J., \& Švaříček, R. (2020). Mandatory home education during the COVID-19 lockdown in the Czech Republic: A rapid survey of 1st-9th Graders' parents. Frontiers in Education, 5(103), 1-8.

Bruining, H., Bartels, M., Polderman, T. J., \& Popma, A. (2020). COVID-19 and child and adolescent psychiatry: An unexpected blessing for part of our population? European Child and Adolescent Psychiatry, 30, 139-140. 
Elo, S., Kääriäinen, M., Kanste, O., Pölkki, T., Utriainen, K., \& Kyngäs, H. (2014). Qualitative content analysis: A focus on trustworthiness. SAGE Open, 4(1), 1-10.

González-Valero, G., Zurita-Ortega, F., Lindell-Postigo, D., Conde-Pipó, J., Grosz, W. R., \& Badicu, G. (2020). Analysis of self-concept in adolescents before and during COVID-19 lockdown: Differences by gender and sports activity. Sustainability, 12(18), 7792-7808.

Jiao, W. Y., Wang, L. N., Liu, J., Fang, S. F., Jiao, F. Y., Pettoello-Mantovani, M., \& Somekh, E. (2020). Behavioral and emotional disorders in children during the COVID-19 epidemic. The Journal of Pediatrics, 221, 264-266.

Lee, J. (2020). Mental health effects of school closures during COVID-19. The Lancet Child and Adolescent Health, 4(6), 421-421.

Lindell-Postigo, D., Zurita-Ortega, F., Ortiz-Franco, M., \& González-Valero, G. (2020). Cross-sectional study of self-concept and gender in relation to physical activity and martial arts in Spanish adolescents during the COVID-19 lockdown. Education Sciences, 10(8), 210-220.

Liu, J. J., Bao, Y., Huang, X., Shi, J., \& Lu, L. (2020). Mental health considerations for children quarantined because of COVID-19. The Lancet Child \&amp; Adolescent Health, 4(5), 347-349.

Mangiavacchi, L., Piccoli, L., \& Pieroni, L. (2021). Fathers matter: Intrahousehold responsibilities and children's wellbeing during the COVID-19 lockdown in Italy. Economics \&amp; Human Biology, 42, 1-19.

Masten, A. S., \& Motti-Stefanidi, F. (2020). Multisystem resilience for children and youth in disaster: Reflections in the context of COVID-19. Adversity and Resilience Science, 1(2), 95-106.

Office of the Children's Commissioner. (2020). Life in Lockdown: Children and young people's views on the nationwide COVID-19 level 3 and 4 lockdown between March and May 2020. Wellington: Retrieved from https://www.occ.org.nz/publications/reports/life-in-lockdown/

Orben, A., Tomova, L., \& Blakemore, S.-J. (2020). The effects of social deprivation on adolescent development and mental health. The Lancet Child \&amp; Adolescent Health, 4(8), 634-640.

Palmer, S., Jenkins, M., Gendall, P., Hoek, J., Beaglehole, B., Bell, C., Williman, J., Rapsey, C., \& Stanley, J. (2020). Psychological distress, anxiety, family violence, suicidality, and wellbeing in New Zealand during the COVID-19 lockdown: A cross-sectional study. PLoS ONE, 15(11), 1-19.

Panda, P. K., Gupta, J., Chowdhury, S. R., Kumar, R., Meena, A. K., Madaan, P., . . Gulati, S. (2021). Psychological and behavioral impact of lockdown and quarantine measures for COVID-19 pandemic on children, adolescents and caregivers: a systematic review and meta-analysis. Journal of Tropical Pediatrics, 67(1), 1-13

Pfeifer, J. H., \& Berkman, E. T. (2018). The development of self and identity in adolescence: Neural evidence and implications for a value-based choice perspective on motivated behavior. Child Development Perspectives, 12(3), 158-164.

Piers, E. V., Shemmassian, S. K., \& Herzberg, D. S. (2018). Piers-Harris Self-Concept Scale, Third Edition (Piers-Harris 3) [Manual]. Western Psychological Services.

Sancho, N. B., Mondragon, N. I., Santamaria, M. D., \& Munitis, A. E. (2021). The Well-being of children in lock-down: Physical, emotional, social and academic impact. Children and Youth Services Review, 127, 1-9.

Shenton, A. K. (2004). Strategies for ensuring trustworthiness in qualitative research projects. Education for Information, 22(2), 63-75.

Singh, S., Roy, D., Sinha, K., Parveen, S., Sharma, G., \& Joshi, G. (2020). Impact of COVID-19 and lockdown on mental health of children and adolescents: A narrative review with recommendations. Psychiatry Research, 293, 1-10.

Strauss, A. L., \& Corbin, J. M. (1990). Basics of Qualitative Research: Grounded Theory Procedures and Techniques. Sage.

Waite, P., Pearcey, S., Shum, A., Raw, J., Patalay, P., \& Creswell, C. (2021). How did the mental health of children and adolescents change during early lockdown during the COVID-19 pandemic in the UK? Journal of Child Psychology and Psychiatry Advances, 1(1), 1-10.

Ybrandt, H. (2008). The relation between self-concept and social functioning in adolescence. Journal of Adolescence, 31(1), 1-16.

Publisher's Note Springer Nature remains neutral with regard to jurisdictional claims in published maps and institutional affiliations. 